A case of TSH secreting pituitary adenoma with Evans' syndrome

Toshiro Seki ${ }^{1}$, Atsushi Yasuda ${ }^{1}$, Natsumi Kitajimaa ${ }^{1}$, Masayuki Oki² Masami Seki ${ }^{3}$, Atsushi Takagi ${ }^{2}$, Masafumi Fukagawa

${ }^{1}$ Division of Nephrology, Endocrinology and Metabolism, ${ }^{2}$ Division of University School of Medicine, ${ }^{3}$ Seirei Numazu Hospital

\begin{tabular}{|c|c|c|c|c|}
\hline \multicolumn{5}{|c|}{ Physical examination } \\
\hline \multicolumn{5}{|c|}{ Height $163.0 \mathrm{~cm}$, Weight $49.0 \mathrm{~kg}$} \\
\hline Vital sign: & \multicolumn{4}{|c|}{$\begin{array}{l}\text { BT } 37.6^{\circ} \mathrm{C}, \mathrm{BP} 182 / 80 \\
\text { RR } 24 / \min \end{array}$} \\
\hline Eyes: & & $\begin{array}{l}\text { Conj. palp: anemic Conj. bulb: icteric } \\
\text { exophthalmos(-) }\end{array}$ \\
\hline Face: & \multicolumn{4}{|c|}{ thickened lips, prognathism of the mandible } \\
\hline Neck: & \multicolumn{4}{|c|}{$\begin{array}{l}\text { thickened lips, prognathism of the mandible } \\
\text { diffusely enlarged thyroid gland }\end{array}$} \\
\hline Chest: & \\
\hline Abdomen: & \multicolumn{4}{|c|}{$\begin{array}{l}\text { soft, no tenderness, normal bowel sound, } \\
\text { hepatosplenomegaly }\end{array}$} \\
\hline Ext.: & \multicolumn{4}{|c|}{ scattered ecchymoses, edema(-) } \\
\hline \multicolumn{5}{|c|}{$\begin{array}{l}\text { She presented with hyperthyroidism symptoms, acromegalic features and } \\
\text { bleeding tendency. }\end{array}$} \\
\hline \multicolumn{5}{|c|}{ Serological findings } \\
\hline & 2660 & & anti nuclear antibody & \\
\hline & & EU & & $<0.3 \quad \mathrm{U} / \mathrm{mL}$ \\
\hline & & & anti & $<5.0 \quad \mathrm{U} / \mathrm{mL}$ \\
\hline & & $\mathrm{IU} / \mathrm{mL}$ & anti SS-1 & $<5.0 \quad \mathrm{U} / \mathrm{mL}$ \\
\hline $\begin{array}{l}\mathrm{C}_{\mathrm{C} 4} \\
\end{array}$ & & $\begin{array}{ll}\mathbf{m g} / \mathbf{d L} \\
\mathbf{m g} / \mathbf{d L}\end{array}$ & tibody & negative \\
\hline TPC & 36 & $\mathrm{IU} / \mathrm{mL}$ & & \\
\hline TgAb & 289 & $\mathrm{IU} / \mathrm{mL}$ & direct & (I) \\
\hline TRAb & & & indirect & $(+)$ \\
\hline
\end{tabular}

The serum levels of C3c and C4 were both decreased, and the antinuclear antibody test was positive. The patient also had an increased sIL-2R, ant
thyroid peroxidase antibody and anti-thyroglobulin antibody. The direct/indirect Coombs' test had a positive result. The impression hematological investigations was hemolytic anemia with thrombocytopenia.

\section{Diagnosis and Treatment}

Diagnosis

Evans' syndrome and hyperthyroidism due to TSHoma with the adenoma concomitantly secreting $\mathrm{GH}$

\section{Treatment}

Steroid treatment for Evans' syndrom

Octreotide treatment and transsphenoidal surgery for the pituitary adenoma

Immediately after admission, immunosuppressive therapy with glucocorticoid
for Evans' syndrome was started. She was also treated with octreotide to control the hyperthyroidism caused by the TSH-secreting adenoma before the operatio The patients' laboratory data, including hemoglobin, platelet, TSH, fT3, fT4, G and IGF-1 levels, were normalized before operation. After surgery, octreotide
treatment was discontinued, and immunosuppressive therapy was maintained The patients' laboratory data, including TSH, fT3, fT4, GH and IGF-1 levels
Ther were normalized, but over the next 5 months, these hormone levels incr.
again. Thus, she was administered a long-acting octreotide treatment again.

\section{Discussion 1}

To the best of our knowledge, there is no case report of Evans' syndrome associated with TSHomas. It would be interesting to determine whether these two diseases exist coincidentally. Recent studies suggested that both diseases have the same immunological mechanism, including insufficient suppressor T-cell activity [1] and TSH receptor autoantibodies [2, 3]. Moreover, it was indicated that hemolytic anemia and thrombocytopenia are the results of thyroid hormone stimulation of the activated reticuloendothelial phagocytic system [4]. In our patient, the hyperthyroidism is due to the TSHoma and is not associated with utoimmune disorders, such as Graves' disease. Thus, we speculate that the excess of thyroid hormone itself might promote autoimmunity in Evans' syndrome.

\section{Concluding remarks}

In summary, we described here a case of TSHoma associated with Evans' syndrome. To the best of our knowledge, there is no case report of Evans' syndrome associated with hyperthyroidism due to TSHoma. Our report suggests that an excess of thyroid hormone itself promotes autoimmunity in Evans' syndrome. Thus, early treatment for hyperthyroidism is necessary in TSHomas because thyroid hormone normalization may prevent the development of Evans' syndrome.
TSH-secreting pituitary adenomas (TSHomas) are rare tumors. These tumors nay lead to the development of hyperthyroidism through a condition called syndrome of inappropriate secretion of TSH (SITSH). In contrast, Evans syndrome is a rare combination of autoimmune hemolytic anemia (AIHA) an idiopathic thrombocytopenic purpura (ITP). A relationship between the pathogenesis of TSHomas and Evans' syndrome is unlikely. However, sever recent cases of Evans' syndrome associated with hyperthyroidism caused by autoimmune thyroid diseases, such as Graves' disease, suggest that these two onditions may have a common immunological mechanism. We report here case of a TSHoma associated with Evans' syndrome. In our case, the hyperthyroidism is due to TSHoma.

\section{Routine laboratory data}

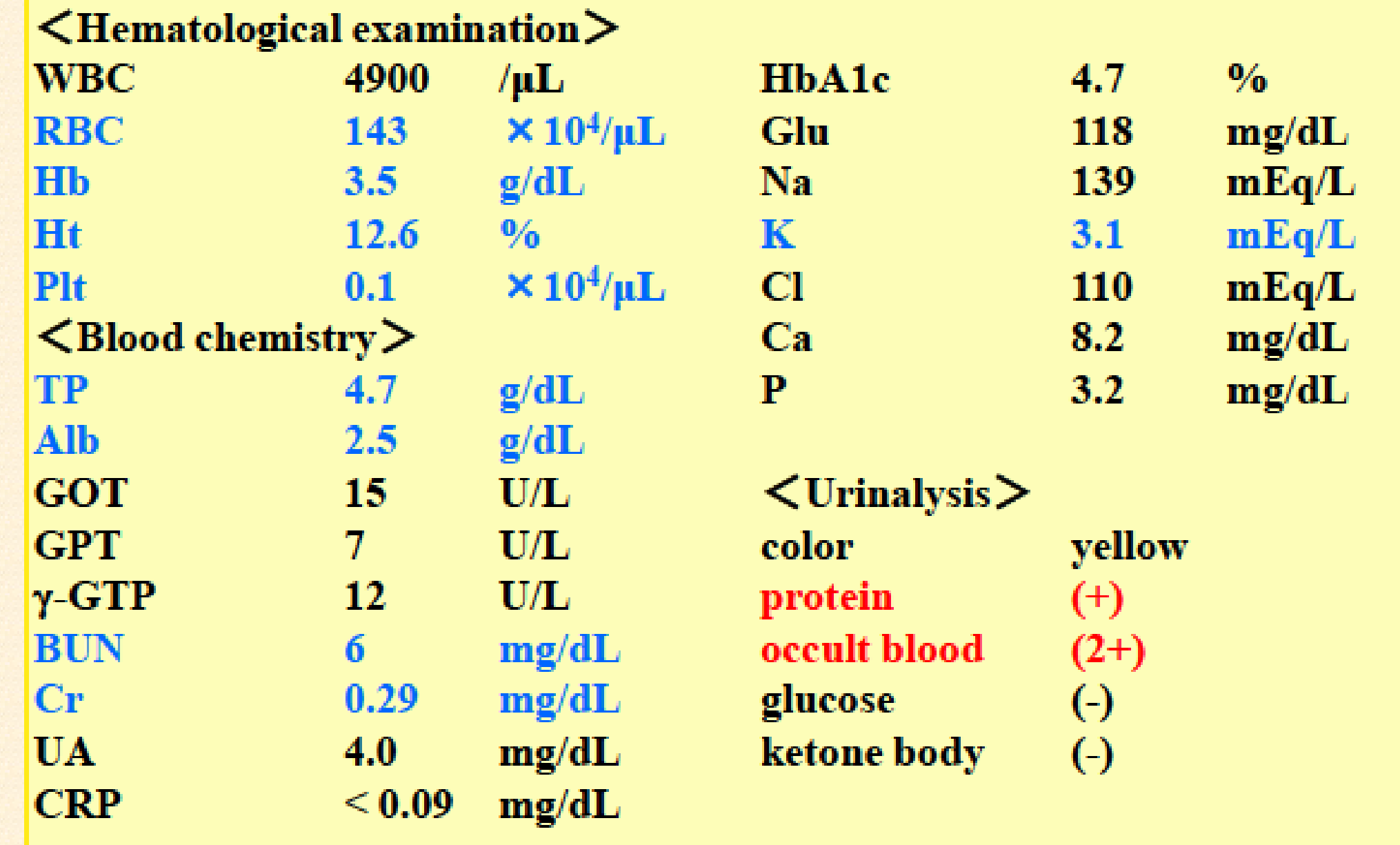

Laboratory results revealed low hemoglobin, hematocrit, red blood cell, Laboratory results revealed low hemoglobin, hematocrit,
and platelet levels. Malnutrition and abnormal urinalysis were observed. MRI findings on admission

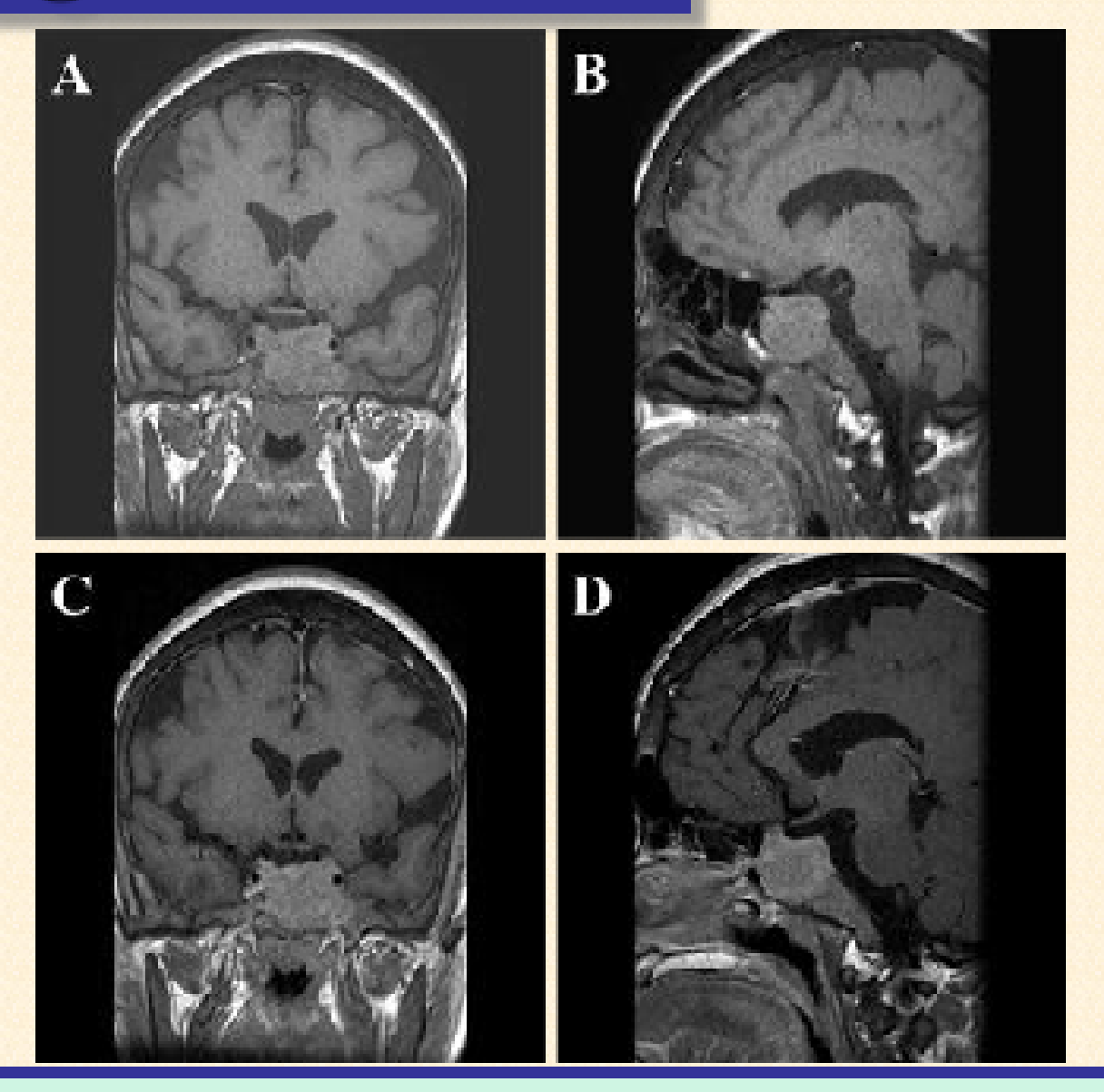
MRI revealed a pituitary tumor of $34 \times 26 \times 28 \mathrm{~mm}$ in the sella involving cavernous
sinuses and extending inferior to the sphenoid sinus (A: unenhanced coronal T1 weighted image, B: unenhanced sagittal T1-weighted image, C: post
coronal T1-weighted image, D: postcontrast sagittal T1-weighted image).

\section{Histopathological findings}
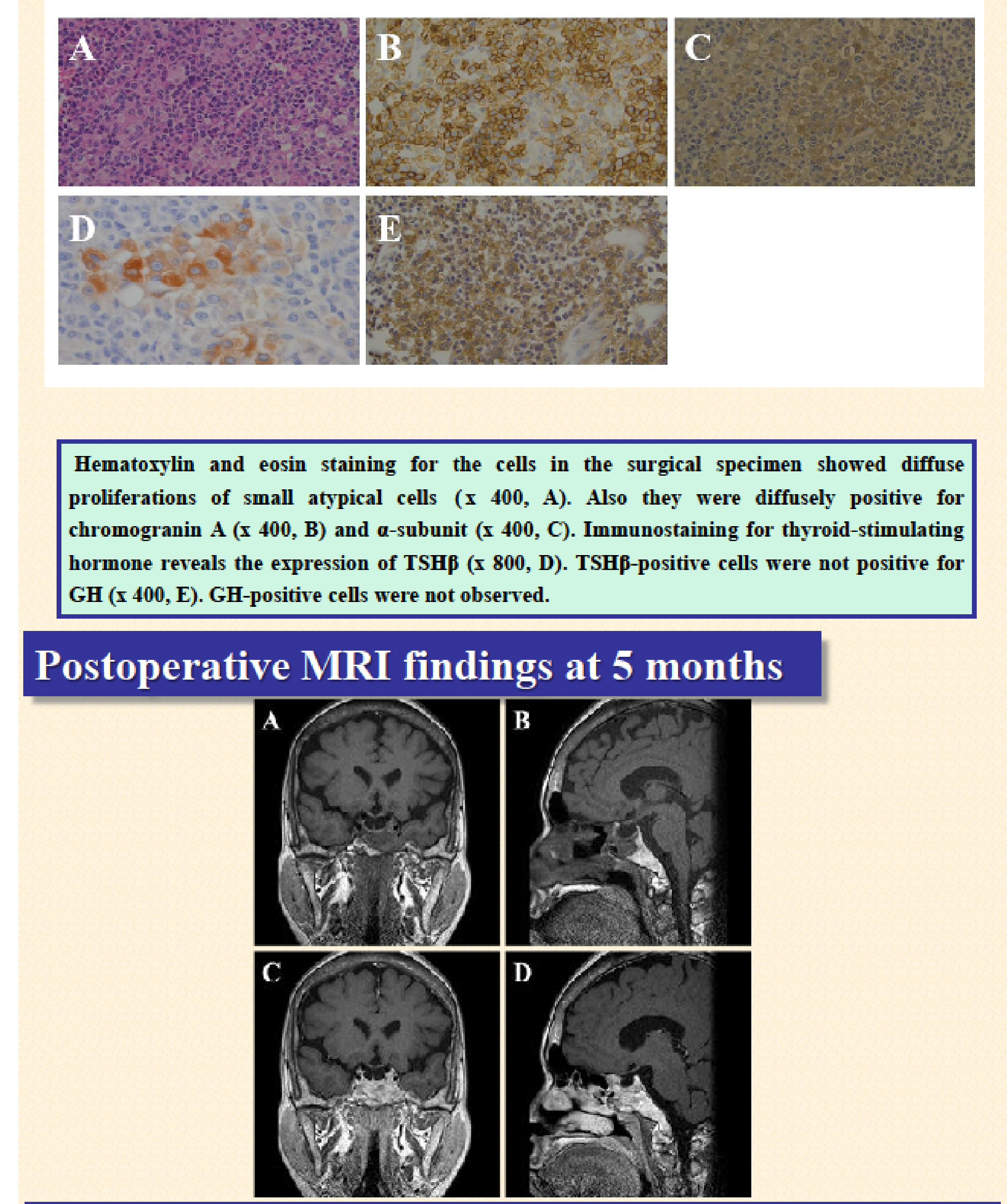

MRI revealed a reduction in the size of the pituitary gland compared with the size found using MRI at admission (A: unenhanced coronal T1-weighted image,
. unenhanced sagittal T1-weighted image, C: postcontrast coronal T1-weighte $\begin{aligned} & \text { B: unenhanced sagittal T1-weighted image, C: postcontrast coronal T1-weighted } \\ & \text { image, D: postcontrast sagittal T1-weighted image). }\end{aligned}$
. Discussion 2

An important question, as described above, concerns the extreme rarity of a coincidence between Evans' syndrome and hyperthyroid states, including Grave's disease and TSHomas. Li et al. speculated that hyperprolactinemia might contribute to the excessive immune response in SLE and investigated patients with SLE and prolactinomas [5]. However, there was no clear relationship between the degree of increase in prolactin levels, hormone effectiveness, and autoimmune disease activity [5]. The authors suggested that these results were due to genetic differences in the reactivity to prolactin [5]. Similarly, among Evans' syndrome patients, there may be a group of patients with a genetic difference that causes susceptibility to the effects of hyperthyroidism.

\section{References}

Nagaratnam N, et al. Haemolytic anaemia, Graves' disease, autoantibodies, case report. Asian J Infectious Dis 1978; 2:271.

Michalek K, Morshed SA, Latif R, D ries TH. TSH reegtor a ibodies.

Sato A, Zakarija M, McKenzie JM. Characteristics of thyrotropin binding to bovine thyroid plasma membranes and the influence of human IgG. Endoc Res Commun 1977; 4:95-113.

Lamberg BA, Kivikangas V, Pelkonen R, Vuopio P. Thrombocytopenia and 3:98-102.

Li M, Keiser HD, Peeva E. Prolactinoma and systemic lupus erythematosus do serum prolactin levels matter? Clin Rheumatol 2006; 25:602-605.

Zhao Z, Zhang M. Pituitary tumor-transforming gene
apoptosis pathway. Cancer Biol Ther 2011; 11:346--348.

Löfgren SE, Frostegarrd J, Truedsson L, Pons-Estel BA, D'Alfonso S, Witte T, et al. Genetic association of miRNA-146a with systemic lupus erythematosus in European
A 30-year-old woman was admitted to a nearby hospital due to purpura and ecchymoses on her limbs and body and epistaxis. There was no family history of notable illness, including autoimmune disease. Laboratory results revealed low hemoglobin, hematocrit, red blood cell, and platelet levels. A hematologic disease was suspected, and she was referred to our hospital. She had a history of malocclusion and thyroid gland enlargement 4 years prior to admission.

\section{Endocrine laboratory data}

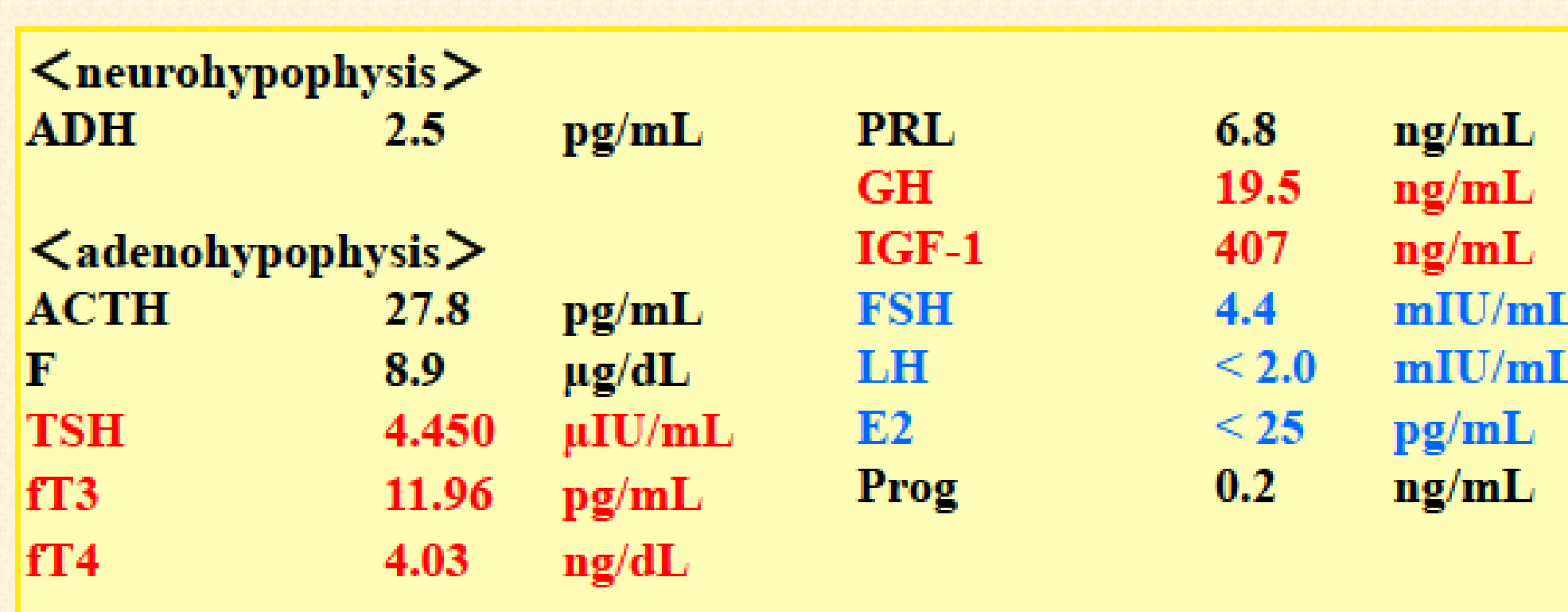

The hormonal examination suggested a syndrome of inappropriate secretio of TSH (SITSH). GH and IGF-1 were also high. The low levels of FSH, LH
(IIT) and E2 implied central hypogonadism.

\section{5gOGTT and octreotide suppression test}

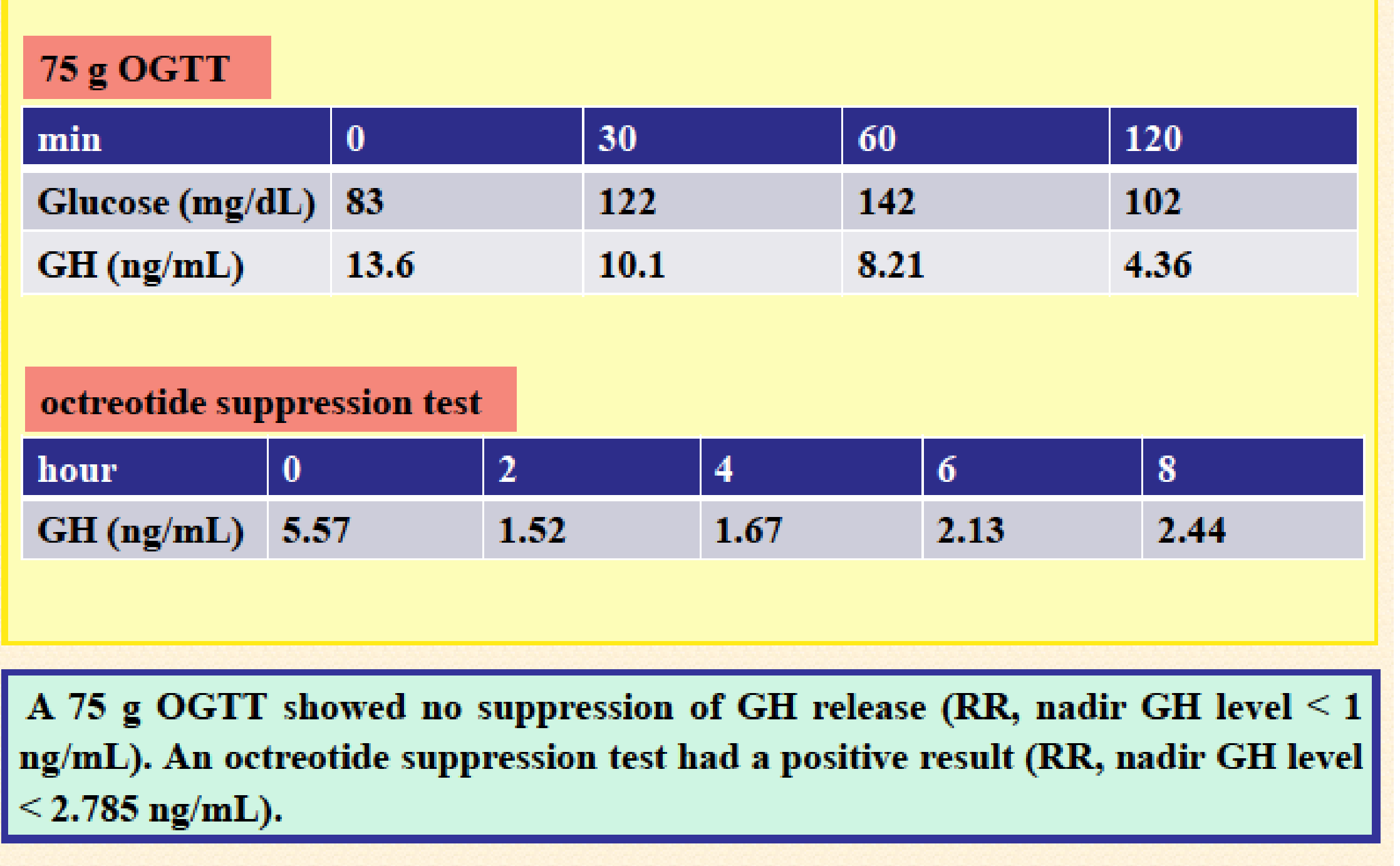

\section{Clinical course of the patient}

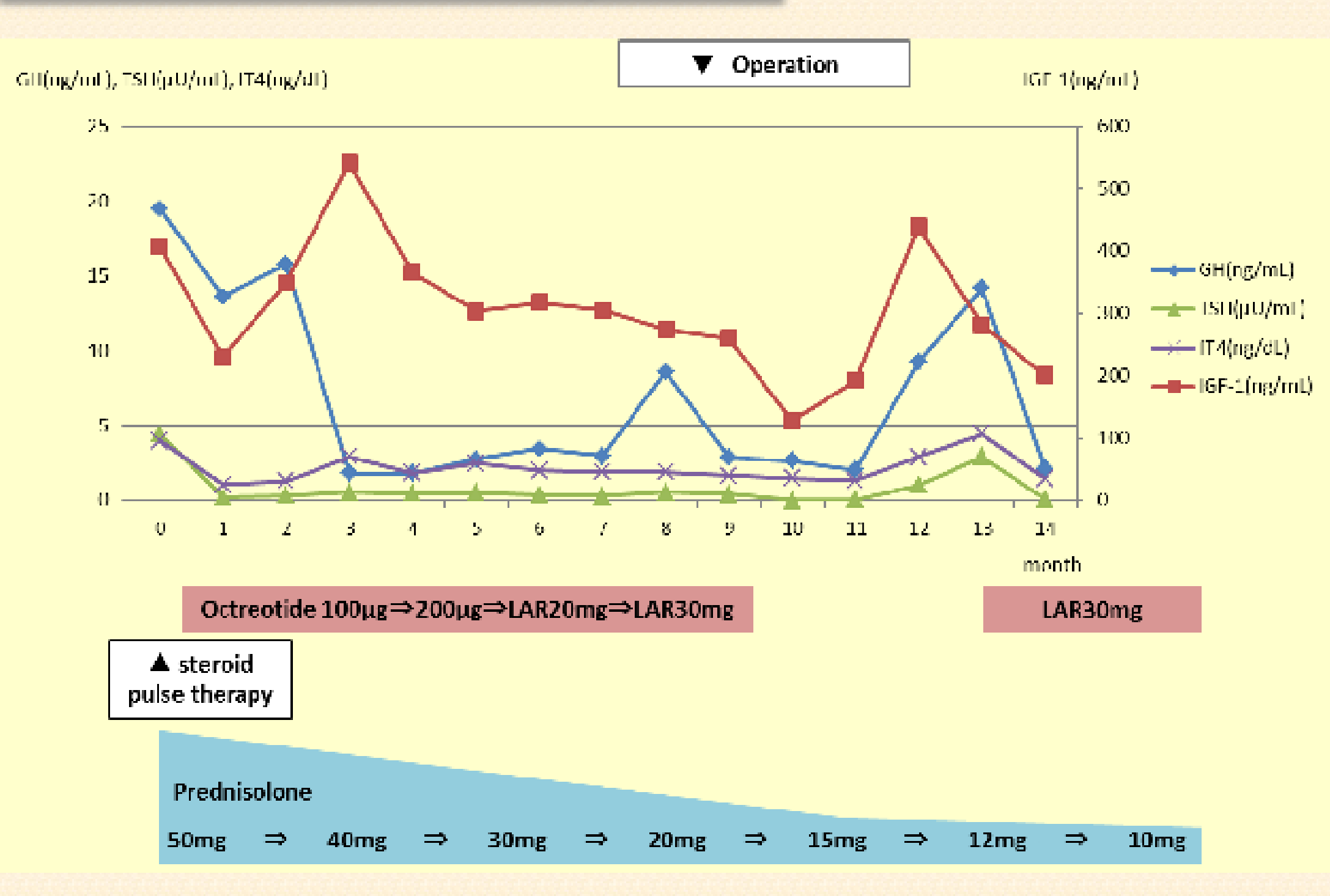

\section{5g OGTT after surgery}

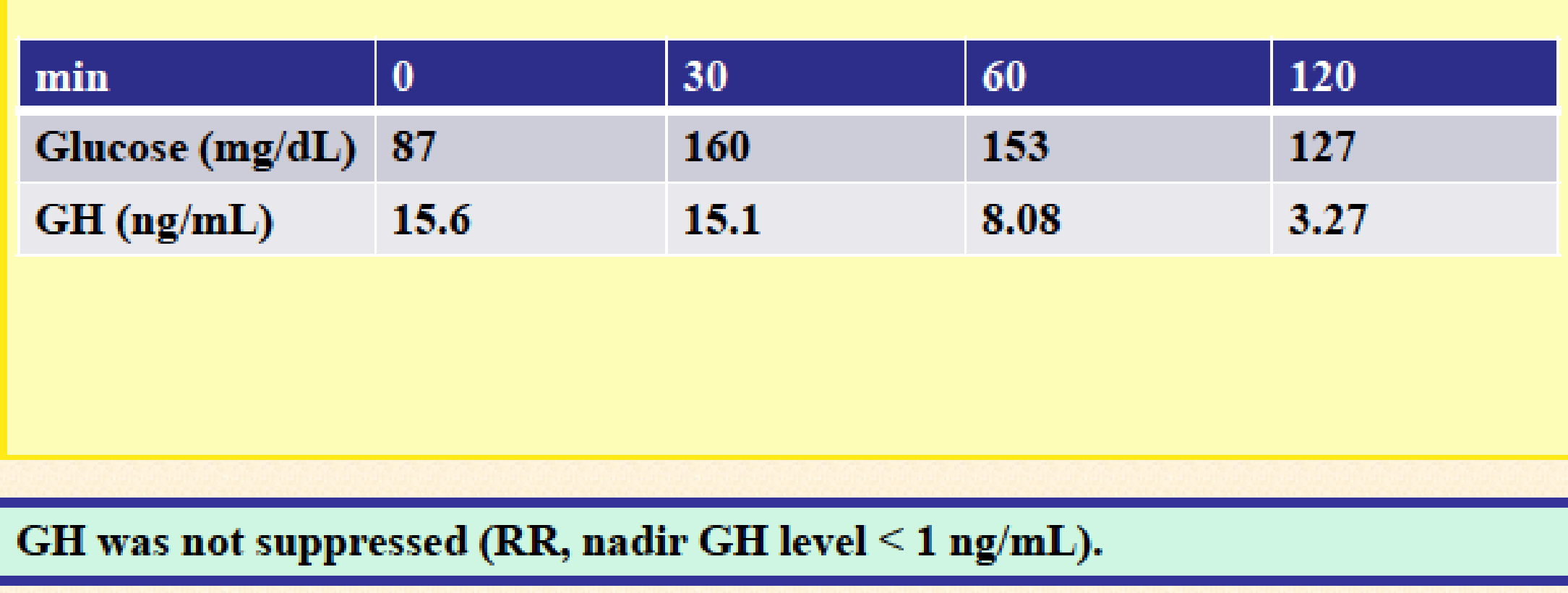

\section{Discussion 3}

Another possible etiology in patients with TSHomas and Evans' syndrome is a enetic polymorphism related to the pituitary tumor-transforming gene (PTTG). PTTG1 overexpression was confirmed to have transforming and tumorigenic activity [6]. In addition, a recent genome-wide study suggested a variant in a region, which was between PTTG1 and the microRNA-146a genes, related to SLE susceptibility [7]. This study statistically analyzed three single-nucleotide polymorphisms (SNPs) in SLE patients [7]. One SNP was genetically associated with SLE and potentially important in SLE etiology [7]. With this in mind, there is a possibility that a pituitary tumor might be related to an autoimmune disease. 